\title{
Percepção de padrões concêntricos em crianças de 8 a 13 Anos
}

\author{
Natanael Antonio dos Santos \\ Valtenice de Cássia Rodrigues de Matos França \\ Éllen Dias Nicácio da Cruz
}

Universidade Federal Federal da Paraiba, João Pessoa-PB, Brasil

Resumo: O objetivo deste trabalho foi mensurar a função de sensibilidade ao contraste de padrões circulares concêntricos (FSCr) com freqüências radiais de 0,$25 ; 0,5 ; 1,0$ e 2,0 cpg em crianças (8-9, 10-11 e 12-13 anos) e adultos jovens. Foram estimados limiares de contraste para 40 participantes (30 crianças e 10 adultos jovens) utilizando o método psicofísico da escolha forçada e nível baixo de luminância. Todos os participantes apresentavam acuidade visual normal e se encontravam livres de doenças oculares identificáveis. Os resultados mostraram que as curvas de FSCr de crianças de 8-9, 10-11 e 12-13 anos melhoram de forma significativa com a idade. Os resultados mostraram ainda que a FSCr de crianças de 12-13 anos é mais baixa do que a de adultos jovens. Estes resultados sugerem que o desenvolvimento da percepção visual de estímulos circulares concêntricos ocorre gradualmente se prolongando além dos 12-13 anos.

Palavras-chave: Sensibilidade ao contraste. Desenvolvimento visual. Crianças. Freqüência radial. Método da escolha forçada.

\section{Perception of concentric patterns in children of 8 to 13 years old}

Abstract: The aim of this study was to measure the function of contrast sensitivity to concentric circular patterns (rCSF) with radial frequencies of $0.25,0.5,1.0$ and $2.0 \mathrm{cpd}$ in children $(8-9,10-$ 11 and 12-13 years of age) and young adults. Contrast thresholds were measured in 40 participants (thirty children and ten young adults) using the psychophysical forced-choice method and low luminance level. All participants were free from identifiable ocular diseases and had normal acuity. The results showed that curves for rCSFs of 8-9, 10-11 and 12-13 years old improved significantly with age. The rCSFs for the 12-13 years old was lower than those of adults. It is suggested that the development of visual perception in concentric circular stimuli increases gradually beyond 12-13 years of age.

Keywords: Contrast sensitivity. Visual development. Children. Radial frequency. Forced-choice staircase method.

\section{Percepción de padrones concéntricos en niños de 8 a 13 Años}

Resumen: El objetivo de este trabajo fue mensurar la función de sensibilidad al contraste de padrones circulares concéntricos (FSCr) con frecuencias radiales de 0,$25 ; 0,5 ; 1,0$ y $2,0 \mathrm{cpg}$ en niños (8-9, 10-11 y 12-13 años) y adultos jóvenes. Fueron estimados umbrales de contraste para 40 participantes (30 niños y 10 adultos jóvenes) utilizando el método psicofísico de la elección forzada y nivel bajo de luminancia. Todos los participantes presentaban acuidad visual normal y estaban libres de enfermedades oculares identificables. Los resultados mostraron que las curvas de la FSCr de niños de 8-9, 10-11 y 12-13 años mejoran de forma significativa con la edad. Los resultados mostraron aún que la FSCr de niños de 12-13 años es más baja que la de adultos jóvenes. Estos resultados sugieren que el desarrollo de la percepción visual de estímulos circulares concéntricos ocurrí gradualmente prolongándose después de los 12-13 años.

Palabras clave: Sensibilidad al contraste. Desarrollo visual. Niños. Frecuencia radial. Método de la elección forzada. 


\section{Introdução}

A mensuração da função de sensibilidade ao contraste (FSC) é considerada uma das técnicas mais completas na avaliação teórica e clínica das funções visuais (Adams \& Courage, 2002; Richman \& Lyons, 1994; Van Sluyters, Atkinson, Held, Hoffman \& Shatz, 1990). Ela pode ser definida como o inverso da curva de limiar de contraste, 1/FSC (Cornsweet, 1970), que corresponde à quantidade mínima de contraste necessária para detectar um objeto qualquer de uma determinada freqüência espacial. Logo, na determinação da FSC, as variáveis experimentais principais são a freqüência espacial (variável independente) definida pelo número de ciclos por grau de ângulo visual (cpg) e o contraste (variável dependente) necessário para detectar a freqüência espacial. $\mathrm{O}$ contraste é a relação entre a diferença da luminância máxima e luminância mínima dividido pela soma das duas.

Pesquisas psicofísicas e eletrofisiológicas com crianças recém-nascidas e em várias faixas etárias destacam a importância da curva de sensibilidade ao contraste (FSC) na investigação do desenvolvimento da percepção visual (Allen, Tyler \& Norcia, 1996; Atkinson, Braddick \& Braddick, 1974; Atkinson, Braddick \& Moar, 1977; Cannon, 1983; Norcia \& Tyler, 1985; Peterzell, Werner \& Kaplan, 1995; Wilson, 1988). Por exemplo, os trabalhos com recémnascidos relatam que a FSC é muito pobre ao nascimento, melhorando rapidamente durante os primeiros meses (Atkinson e cols., 1977; Allen e cols., 1996; Bradley \& Freeman, 1982; Kelly, Borchert \& Teller, 1997; Peterzell e cols., 1995). Já os trabalhos com crianças a partir da idade pré-escolar mostram que a FSC para grade senoidal melhora gradativamente à medida que a criança se desenvolve (Adams \& Courage, 2002; Allen e cols., 1996; Arundale, 1978; Atkinson e cols., 1974; Benedek, Benedek, Kéri \& Janáky, 2003; Bradley \& Freeman, 1982; Ellemberg, Lewis, Liu, \& Maurer, 1999; Peterzell e cols., 1995; Richman \& Lyons, 1994). No entanto, existem diferenças entre os resultados destes estudos quanto ao período em que a criança alcança a performance visual do adulto. Por exemplo, Bradley e Freeman (1982) mensuraram a FSC para crianças de 2 a 16 anos, utilizando o método psicofísico da escolha forçada e luminância média de $250 \mathrm{~cd} / \mathrm{m}^{2}$. Os resultados mostraram que as crianças atingem a FSC dos adultos por volta dos 8 anos de idade.

Por sua vez, Ellemberg e cols. (1999) mediram a FSC para crianças de 4 a 7 anos e adultos de 18 a 26 anos, utilizando o método psicofísico dos limites e estímulos de grade senoidal com freqüências espaciais de 0,$33 ; 0,5 ; 1,2,3,5,10$ e 20 cpg e com luminância média de $9 \mathrm{~cd} / \mathrm{m}^{2}$. Os autores não encontraram diferenças significativas entre a FSC de crianças de 7 anos e adultos.

Por outro lado, Adams e Courage (2002) mediram a FSC para crianças de 1 mês a 9 anos, utilizando o método psicofísico do olhar preferencial e estímulos de grade senoidal com freqüências espaciais de 0,$4 ; 0,8 ; 1,6 ; 3,2$ ou 4,8 cpg e com luminância média de $70 \mathrm{~cd} / \mathrm{m}^{2}$. Os resultados mostraram que a FSC das crianças desenvolve-se rapidamente até os três anos de idade, depois disto continua desenvolvendo-se mais lentamente, atingindo, a FSC dos adultos por volta dos 9 anos.

Finalmente, Benedek e cols. (2003) mensuraram a FSC de crianças e adolescentes de 5 a 14 anos, utilizando o método do ajuste e estímulos de grade senoidal, estáticos $(0 \mathrm{~Hz})$ e dinâmicos $(8 \mathrm{~Hz})$, com as freqüências espaciais de 0,$5 ; 1,2 ; 1,9 ; 5,7 ; 7,2$ e 14,3 cpg em dois níveis de luminância, 0,09 e 9 cd/ $\mathrm{m}^{2}$. Os dados mostraram que as crianças só atingiram a FSC dos adultos por volta dos 11-12 anos.

Em termos gerais, estes estudos mostram que a FSC de crianças para grade senoidal atinge o padrão do adulto por volta dos 7 a 12 anos. Esta variabilidade pode estar relacionada aos métodos e as condições experimentais empregadas que variaram muito entre os trabalhos.

No presente estudo, caracterizou-se curvas de sensibilidade ao contraste para estímulos circulares 
concêntricos de freqüências radiais (FSCr) em crianças de 8 a 13 anos e adultos jovens (19-22 anos), utilizando o método psicofísico da escolha forçada e nível mesópico de luminância $\left(0,7 \mathrm{~cd} / \mathrm{m}^{2}\right)$. Os objetivos do presente estudo foram determinar a sensibilidade ao contraste para padrões circulares simétricos de freqüências radiais (FSCr) e investigar a existência de relações entre o desenvolvimento visual e as estimativas de sensibilidade ao contraste para estímulos visuais elementares com freqüências radiais circularmente simétricas.

Em sua maioria, as pesquisas que procuraram determinar e relacionar a FSC ao desenvolvimento das funções visuais ou da percepção visual de contraste foram realizadas com padrões espaciais do tipo grade senoidal vertical e níveis fotópicos de luminância (Adams \& Courage, 2002; Arundale, 1978; Bradley \& Freeman, 1982; Benedek e cols., 2003; Ellemberg e cols., 1999). As pesquisas que visaram determinar a resposta do sistema visual humano para freqüências radiais são dispersas e raras com adultos e idosos (Kelly, 1982; Kelly \& Magnuski, 1975; Santos, Oliveira, Nogueira \& Simas, 2006; Simas \& Santos, 2002) e não encontramos nenhum estudo na literatura que relacionasse a sensibilidade ao contraste para freqüências radiais (ou com configurações semelhantes) ao desenvolvimento visual da criança.

Pesquisas dessa natureza são de grande relevância tanto do ponto de vista da pesquisa básica quanto da aplicação clínica, pois os valores de contraste obtidos podem ser utilizados como valores normativos para avaliar o desenvolvimento visual de crianças saudáveis, além de contribuir para o diagnóstico de possíveis problemas na visão de contraste em crianças com patologias neurofisiológicas e/ou visuais.

\section{Método}

\section{Participantes}

Participaram do estudo 40 voluntários entre crianças e adultos de ambos os sexos. Os partici- pantes foram distribuídos igualmente em quatro grupos [8-9 anos $(\mathrm{N}=10 ; \mathrm{M}=8,74 ; \mathrm{DP}=0,77), 10-11$ $\operatorname{anos}(\mathrm{N}=10 ; \mathrm{M}=10,73 ; \mathrm{DP}=0,46), 12-13 \operatorname{anos}(\mathrm{N}=10$; $\mathrm{M}=12,95$; $\mathrm{DP}=0,57)$ e adultos jovens de 19-22 anos $(\mathrm{N}=10 ; \mathrm{M}=21,3$ anos; $\mathrm{DP}=13)]$. Os símbolos $M \mathrm{e}$ $D P$ representam à idade média (ano, mês) e o desvio padrão (mês) de cada grupo, respectivamente. Todos apresentavam acuidade visual normal ou corrigida e não tinham história de patologia ocular. A participação na pesquisa ocorreu mediante assinatura de Termo de Consentimento Livre e Esclarecido conforme a Resolução no 196/96 do Conselho Nacional de Saúde (Ministério da Saúde), que trata das diretrizes e normas de pesquisas envolvendo seres humanos. No caso dos menores de 21 anos, o termo foi assinado pelo responsável. O projeto foi cadastrado na Comissão Nacional de Ética em Pesquisa do Ministério da Saúde (CONEP/MS-064608) e aprovado pelo Comitê de Ética do Centro de Ciências da Saúde.

\section{Equipamentos e Estímulos}

Os estímulos radiais foram gerados em um monitor de vídeo CLINTON MEDICAL monocromático de 21 polegadas, digital e de alta resolução controlado por um microcomputador. Uma cadeira foi fixada a $150 \mathrm{~cm}$ da tela do monitor de vídeo. A luminância média foi de $0,7 \mathrm{~cd} / \mathrm{m}^{2}$ ajustada por um fotômetro do tipo SPOT METTER, com precisão de um grau, ASAHI PENTAX. A luminância mínima foi de $0,5 \mathrm{~cd} / \mathrm{m}^{2}$ e a máxima de $0,9 \mathrm{~cd} / \mathrm{m}^{2}$. $O$ ambiente do laboratório era cinza para melhor controle da luminância.

Foram utilizados estímulos circularmente simétricos com freqüências radiais de 0,$25 ; 0,5 ; 1$ e 2 ciclos por grau de ângulo visual, cpg (Figura 1). Estes eram circulares com um diâmetro de 7 graus de ângulo visual a $150 \mathrm{~cm}$ de distância da tela e foram gerados em tons de cinza e apresentados em tempo real no monitor. A utilização de freqüências espaciais mais baixas ocorreu devido ao baixo nível de luminância utilizado, pois a curva de sensibilidade ao 
contraste é deslocada para as freqüências espaciais baixas (G. Benedek e cols., 2003).
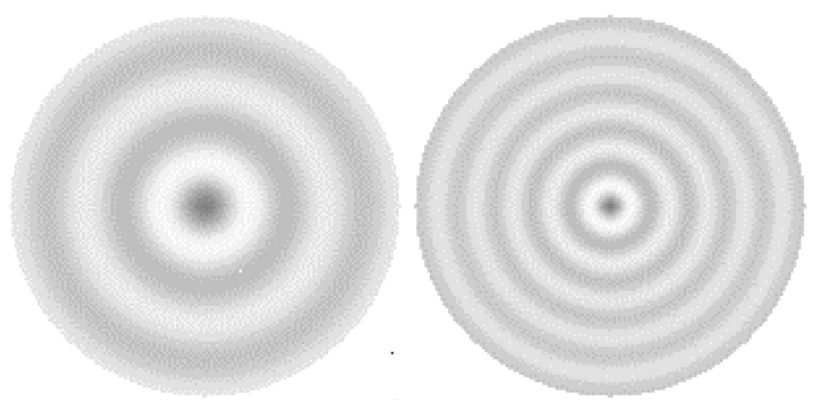

Figura 1. Exemplos de estímulos de freqüências radiais, à esquerda $1,0 \mathrm{cpg}$ e à direita 2,0 cpg. Estímulos originalmente calibrados para serem vistos a $150 \mathrm{~cm}$ de distância.

A primeira proposta de estudar padrões radiais definidos em coordenadas polares em vez de coordenadas cartesianas (p.ex., grades senoidais) partiu de Kelly (1960), que sugeriu este tipo de estímulo circular concêntrico modulado pela função cilíndrica de Bessel $\left(\mathrm{J}_{0}\right)$, onde a freqüência espacial varia ao longo do raio. Contrário da grade senoidal, os estímulos radiais têm um centro de fixação bem definido (Figura 1). Outras informações sobre estes estímulos podem ser encontradas na literatura (Kelly, 1960, 1982; Kelly \& Magnuski, 1975; Santos \& Simas, 2002; Santos e cols., 2006; Simas \& Santos, 2002).

\section{Procedimento}

Utilizou-se um delineamento experimental com medidas repetidas como é peculiar às pesquisas em percepção visual com método psicofísico. Neste tipo de delineamento, cada grupo passa por mais de uma ou todas as condições em momentos e ordens diferentes. $\mathrm{O}$ efeito da ordem foi controlado pela técnica de contrabalanceamento. $\mathrm{O}$ delineamento experimental foi composto por uma variável dependente (limiar de contraste) e por duas variáveis independentes: freqüência radial em quatro níveis $(0,25 ; 0,5 ; 1$ e $2 \mathrm{cpg})$ e faixa etária em três níveis (8-9, 10-11 e 12-13 anos).
As estimativas foram realizadas com o método psicofísico da escolha forçada (Santos, Simas \& Nogueira, 2004; Santos e cols., 2006; Wetherill \& Levitt, 1965). Este método se baseia no cálculo da probabilidade de acertos consecutivos por parte do participante, ou seja, em cerca de 100 apresentações de escolhas entre os dois estímulos, a freqüência radial (estímulo de teste) é percebida $79 \%$ das vezes pelo voluntário. $\mathrm{O}$ procedimento para medir o limiar para cada freqüência consistiu na apresentação sucessiva simples do par de estímulos e o participante teve que escolher dentre eles qual continha a freqüência radial. $\mathrm{O}$ outro estímulo (estímulo neutro) foi sempre um padrão homogêneo com luminância média de $0,7 \mathrm{~cd} /$ $\mathrm{m}^{2}$. O critério adotado para variar o contraste de cada freqüência radial testada foi o de três acertos consecutivos para decrescer uma unidade e um erro para acrescer da mesma unidade $(0,08 \%)$.

Durante cada sessão experimental foi apresentada uma seqüência de estímulos iniciada com um sinal sonoro acompanhado imediatamente pela apresentação do primeiro estímulo por $2 \mathrm{~s}$, seguido de um intervalo entre estímulos de 1 s e pela apresentação do segundo estímulo por $2 \mathrm{~s}$ e da resposta do participante. A ordem de apresentação dos estímulos era aleatória. Se a resposta do voluntário fosse correta, era seguida por outro sinal sonoro e um intervalo de $3 \mathrm{~s}$ para a seqüência se repetir. $\mathrm{O}$ sinal sonoro que indicava o início da apresentação do par de estímulos e o que indicava a escolha correta eram diferentes. A duração da sessão experimental variou entre 5 e 10 minutos, dependendo dos erros e acertos do participante até proporcionarem um total de seis reversões (ou três valores mínimos e três máximos de contraste), conforme requerido para o final automático da mesma.

Cada uma das freqüências da curva de sensibilidade ao contraste foi estimada pelo menos duas vezes (duas sessões experimentais), em dias diferentes, por cada um dos participantes. Em média, vinte curvas foram mensuradas para cada grupo de voluntários totalizando 80 sessões experimentais. 
Todas as medições ocorreram à distância de $150 \mathrm{~cm}$, com visão binocular.

Os participantes foram instruídos a pressionar o botão do lado esquerdo do mouse quando a freqüência radial era apresentada primeiro e o botão do lado direito quando era apresentada em segundo lugar, isto é, após o estímulo neutro. Os experimentos só começaram quando o experimentador certificouse que os participantes entenderam e responderam conforme as instruções. É importante mencionar que cada sessão foi iniciada com o estímulo teste com contraste em nível supralimiar, isto é, 0,12 para todas as freqüências radiais.

Após cada sessão, o programa produziu uma folha de resultados com a situação experimental e os seis valores de contraste obtidos pelas reversões. Os valores de contraste obtidos para cada freqüência foram agrupados em planilhas por faixa etária e a grande média foi utilizada como estimativa do limiar de contraste (ou sensibilidade ao contraste) em função da freqüência radial. Os mesmos valores de contraste agrupados em planilhas, por condição (frequêencia e faixa etária), também foram utilizados para as análises estatísticas (ANOVA para medidas repetidas e post-hoc Tukey HSD).

\section{Resultados}

A Figura 2 mostra curvas de FSCr (função de sensibilidade ao contraste para freqüências radiais) para cada uma das quatro faixas etárias (crianças de 8-9, 10-11, 12-13 e adultos de 19-22 anos). As curvas foram traçadas a partir da média geral de cada grupo para cada freqüência. Os limiares de contraste são apresentados em função da freqüência radial. A sensibilidade ao contraste (FSCr) é o inverso do limiar de contraste (1/FSCr). Isto é, quanto menor o limiar de contraste maior a sensibilidade do sistema visual humano e vice-versa. Assim, os menores valores de limiares correspondem aos maiores valores de sensibilidade ao contraste.

A sensibilidade máxima ocorreu na freqüência radial de $0,25 \mathrm{cpg}$ para todas as idades (Figura 2).

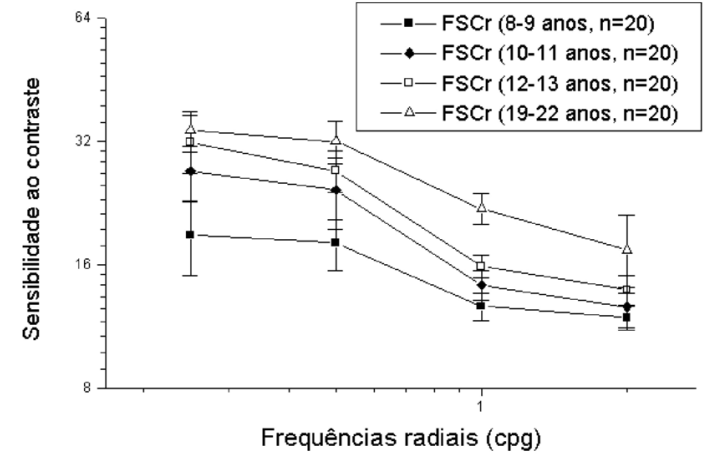

Figura 2. Curvas de sensibilidade ao contraste de crianças de 8-9, 10-11 e 12-13 anos e adultos jovens (19-22 anos) para freqüências radiais (FSCr). $\mathrm{O}$ " $\mathrm{n}$ " representa o número de curvas mensuradas para cada faixa etária. As linhas verticais mostram o erro padrão da média para cada freqüência $(0,25 ; 0,5 ; 1$ e 2 cpg $)$.

Entretanto, a diferença na sensibilidade ao contraste na faixa de máxima sensibilidade (freqüência de $0,25 \mathrm{cpg}$ ) entre as crianças de 8-9 e 1011 anos e 10-11 e 12-13 anos foi da ordem de 1,43 e 1,19 vezes, respectivamente. Seguem entre parênteses os valores de limiar de contraste obtidos por cada grupo (média geral) na freqüência de $0,25 \mathrm{cpg}$ : 8-9 anos $(0,0530), 10-11$ anos $(0,0370)$ e 12-13 anos $(0,0314)$. Observa-se que a sensibilidade das crianças melhora com o aumento da idade, pois as crianças precisaram de menos contraste para detectar a freqüência radial de $0,25 \mathrm{cpg}$. Os resultados para as outras freqüências radiais se comportaram de forma semelhante (Figura 2).

A ANOVA para medidas repetidas revelou diferenças significativas entre as idades, $\mathrm{F}_{(3,620)}=$ $173,89(\mathrm{p}<0,01)$, entre as freqüências radiais, $\mathrm{F}_{(3,1860)}$ $=663,26(p<0,01)$, e uma interação entre freqüências radiais e idades, $\mathrm{F}_{(9,1860)}=7,61(\mathrm{p}<0,01)$. As análises com o teste post-hoc Tukey HSD mostraram diferenças estatísticas significantes na sensibilidade ao contraste ( $\mathrm{FSCr}$ ) entre as crianças em todas freqüências $(p<0,01)$ e entre crianças $12-13$ anos e adultos jovens em todas as freqüências $(p<0,01)$. Estes resultados mostram que a $\mathrm{FSCr}$ melhora de forma 
significativa com o aumento da idade e que a $\mathrm{FSCr}$ de crianças 12-13 anos é diferente da FSCr de adultos. Isto é, a maturação da FSCr se estende para idades maiores do que aquelas utilizadas neste estudo.

\section{Discussão}

A proposta de determinar a resposta do sistema visual de crianças (8-9, 10-11 e 12-13 anos) e adultos (19-22 anos) para padrões de freqüências radiais circularmente simétricos focaliza algumas questões básicas relacionadas ao desenvolvimento da percepção visual de objetos e contraste em crianças. Mostra, por exemplo, o comportamento da FSCr para crianças de 8 a 13 anos, especificando a faixa de freqüência radial que o sistema visual detecta melhor (máxima sensibilidade ao contraste) em nível baixo de luminância e o processo de maturação do sistema visual para este tipo de estímulo.

Os resultados mostraram que a faixa de maior sensibilidade para estímulos radiais (FSCr) ocorreu na freqüência mais baixa de $0,25 \mathrm{cpg}$ para todas as idades (Figura 2). Isto significa que embora o desempenho do sistema visual da criança para processar este tipo de padrão melhore com o aumento da idade, a faixa de freqüência radial que o sistema visual é mais sensível continua em $0,25 \mathrm{cpg}$. Estes dados obtidos com luminância baixa ou mesópica $(0,7$ $\mathrm{cd} / \mathrm{m}^{2}$ ) são diferentes de outros na literatura com luminância média acima de $9 \mathrm{~cd} / \mathrm{m}^{2}$ (Kelly, 1982; Kelly \& Magnuski, 1975; Simas, Santos \& Thiers, 1997) que encontraram faixa de máxima sensibilidade para freqüências radiais entre 0,5-2 cpg. O deslocamento da sensibilidade máxima para freqüências mais baixas $(0,25 \mathrm{cpg})$ já era esperado e pode estar relacionado tanto ao funcionamento da via visual parvocelular quanto da magnocelular que estão ativas em condições de luminância mesópica (Benedek e cols., 2003; Ellemberg e cols., 1999). Pois, estudos discutem que o processamento visual da forma e contraste envolve pelo menos dois sistemas: a via visual parvocelular que é especializada no processamento de freqüencias espaciais médias e altas ou detalhes finos e opera em níveis altos ou fotópicos de luminância e a via visual magnocelular que é especializada no processamento de freqüências espaciais baixas e opera em níveis baixos ou escotópicos de luminância (Banks \& Bennett, 1988; Brown, Dobson \& Maier, 1987; Ellemberg, Lewis, Maurer \& Brent, 2000; Hickey, 1977; Kiorpes \& Movshon, 1998; Van Sluyters e cols., 1990; Wilson, 1988; Yuodelis \& Hendrickson, 1986).

Quanto ao processo de maturação do sistema visual para padrões radiais, observa-se que os perfis gerais das curvas de FSCr de crianças de 8-9, 10-11 e 12-13 anos são semelhantes entre si e entre a FSCr de adultos jovens (19-22 anos). Entretanto, os resultados mostram aumentos significantes na $\mathrm{FSCr}$ na comparação entre todas as idades $(p<0,01)$. Aumento na sensibilidade ao contraste relacionado ao desenvolvimento já era esperado, pois vários estudos relatam dados nesta direção com métodos psicofísicos diferentes só que utilizando grade senoidal vertical (Adams \& Courage, 2002; Arundale, 1978; Bradley \& Freeman, 1982; Benedek e cols., 2003; Ellemberg e cols, 1999). Estudos relacionando estímulos radiais, FSCr e desenvolvimento não foram encontrados na literatura.

Estes resultados mostram ainda que o desempenho (FSCr) das crianças é estatisticamente diferente do desempenho de adultos $(\mathrm{p}<0,01)$. Por exemplo, as crianças de 12-13 anos foram as que mais se aproximaram do desempenho dos adultos, ainda assim, elas precisaram em média da ordem 1,07; 1,18; 1,38 e 1,25 vezes mais contraste do que os adultos para detectar as freqüências radiais de 0,$25 ; 0,5 ; 1$ e 2 cpg, respectivamente. As diferenças foram maiores nas freqüências radiais de 1 e 2 cpg (Figura 2). Estes achados com freqüências radiais (FSCr) são diferentes daqueles encontrados na literatura para grades senoidais verticais (FSC) que relatam que a FSC das crianças atinge o desempenho dos adultos antes dos 12 anos (Adams \& Courage, 2002; Allen e cols., 1996; Arundale, 1978; Atkinson e cols., 1974; Benedek e cols., 2003; Bradley \& Freeman, 1982; Ellemberg e cols., 1999; Peterzell e cols., 1995). Entretanto, esta é uma comparação indireta, pois estes autores 
utilizaram estímulos espaciais (grade senoidal) que são diferentes dos estímulos de freqüências radiais utilizados neste estudo. Grade senoidal é um estímulo de freqüência espacial clássico cuja modulação de amplitude de luminância varia, de acordo com o seno e/ou co-seno, em uma direção no espaço e pode ser facilmente definido em um sistema de coordenadas cartesianas. Ao passo que o estímulo de freqüência radial é um padrão cuja modulação de amplitude de luminância varia, de acordo com o seno ou o co-seno, na direção radial (ou seja, a luminância varia do centro para a periferia nesse tipo de padrão), o que o torna um estímulo facilmente definido em um sistema de coordenadas polares com a origem do sistema de coordenadas no centro do estímulo (Kelly, 1960; Simas $\&$ Santos, 2002). As vantagens de estímulos radiais são que os mesmos apresentam um centro de fixação central bem definido (Figura 1) e, segundo alguns autores, são naturalmente mais apropriados para estudar o sistema visual con-siderando a simetria aproximadamente circular da retina (Kelly, 1960; Kelly \& Magnuski, 1975). Além disto, estudos psicofísicos (Santos \& Simas, 2002; Santos e cols., 2004, 2005; Simas \& Santos, 2002; Wilson \& Wilkinson, 1998; Wilson, Wilkinson \& Asaad, 1997) e neurofisiológicos (Gallant, Connor, Rakshit, Lewis \& Van Essen, 1996; Heywood, Gadotti \& Cowey, 1992; Merigan, 1996; Wilkinson e cols, 2000) sugerem que grade senoidal e padrão radial são processados por áreas visuais distintas. Isto é, estímulos visuais do tipo grade senoidal em coordenadas cartesianas são processados na área visual V1 (córtex visual primário), enquanto estímulos visuais de freqüências radiais em coordenadas polares são processados por áreas visuais extra-estriados V4 e córtex ínferotemporal (IT).

Em termos gerais, os resultados mostraram que a FSCr varia em função do desenvolvimento e que estudos com padrões espaciais com configurações diferentes podem determinar quais estímulos são mais adequados para caracterizar a resposta do sistema visual. Podem, ainda, demonstrar de que maneira o processo de maturação da curva de sensibilidade ao contraste se relaciona com o desenvolvimento das áreas visuais ou vias sensoriais. Isto, considerando a hipótese que estímulos em coordenadas cartesianas e polares são processados por áreas visuais distintas (Gallant e cols., 1996; Heywood e cols., 1992; Merigan, 1996; Wilson \& Wilkinson, 1998; Wilkinson e cols., 2000). Por outro lado, este estudo se limitou em estudar freqüências radiais baixas em nível baixo de luminância ou mesópica. Novas pesquisas, comparando estímulos radiais e grades senoidais com níveis de luminâncias e freqüências altas, precisam ser realizadas para maior discernimento empírico. É nesta perspectiva que prosseguiremos com as nossas investigações

Finalmente, estudo desta natureza permite uma melhor compreensão do desenvolvimento sensorial de crianças, contribuindo para várias áreas da Psicologia, como por exemplo, a Psicologia do Desenvolvimento, Psicologia Experimental, Psicologia Sensorial, Neurociências, etc. Além disto, este estudo mostra que é possível integrar pesquisa básica e aplicada, já que os resultados podem ser utilizados como parâmetros de avaliação clínica de crianças com problemas visuais e/ou neurofisiológicos.

\section{Referências}

Adams, R. J., \& Courage, M. L. (2002). Using a single test to measure human contrast sensitivity from early childhood to maturity. Vision Research, $42,1205-1210$

Allen, D., Tyler, C. W., \& Norcia, A. M. (1996). Development of grating acuity and contrast sensitivity in the central and peripheral visual field of the human infant. Vision Research, 36, 19451953.

Arundale, K. (1978). An investigation into the variation of human contrast sensitivity with age and ocular pathology. British Journal of Ophthalmology, 62, 213-215.

Atkinson, J., Braddick, O., \& Braddick, F. (1974). Acuity and contrast sensivity of infant vision. Nature, 247, 403-404. 
Atkinson, J., Braddick, O., \& Moar, K. (1977). Contrast sensitivity of the human infant for moving and static patterns. Vision Research, 17, 1045-1047.

Banks, M. S., \& Bennett, P. J. (1988). Optical and photoreceptor immaturities limit the spatial and chromatic vision of human neonates. Journal of the Optical Society of America A, Optics and Image Science, 5, 2059-2079.

Benedek, G., Benedek, K., Kéri, S., \& Janáky, M. (2003). The scotopic low-frequency spatial contrast sensitivity develops in children between the ages of 5 and 14 years. Neuroscience Letters, $345,161-164$.

Bradley, A., \& Freeman, R. D. (1982). Contrast sensitivity in children. Vision Research, 22, 953-959.

Brown, A. M., Dobson, V., \& Maier, J. (1987). Visual acuity of human infants at scotopic, mesopic, and photopic luminances. Vision Research, 27, 1845-1858.

Cannon, M. W., Jr (1983). Contrast sensitivity: Psychophysical and evoked potential methods compared. Vision Research, 23, 87-95.

Cornsweet, T. N. (1970). Visual perception. New York: Academic Press.

Ellemberg, D., Lewis, T. L., Liu, C. H., \& Maurer, D. (1999). Development of spatial and temporal vision during childhood. Vision Research, 39, 2325-2333.

Ellemberg, D., Lewis, T. L., Maurer, D., \& Brent, H. P. (2000). Influence of monocular deprivation during infancy on the later development of spatial and temporal vision. Vision Research, 40, 3283-3295.

Gallant, J. L., Connor, C. E., Rakshit, S., Lewis, J. W., \& Van Essen, D. C. (1996). Neural responses to polar, hyperbolic, and cartesian gratings in area V4 of the macaque monkey. Journal of Neurophysiology, 76, 2718-2739.
Heywood, C. A., Gadotti, A., \& Cowey, A. (1992). Cortical area V4 and its role in the perception of color. Journal of Neuroscience, 12, 4056-4065.

Hickey, T. L. (1977). Postnatal development of the human lateral geniculate nucleus: Relationship to a critical period for the visual system. Science, 198, 836-838.

Kelly, D. H. (1960). J stimulus patterns for visual research. Journal of the Optical Society of America, 50, 1115-1116.

Kelly, D. H. (1982). Motion and vision: IV. Isotropic and anisotropic spatial responses. Journal of the Optical Society of America, 72, 432-439.

Kelly, J. P., Borchert, K., \& Teller, D. Y. (1997). The development of chromatic and achromatic contrast sensitivity in infancy as tested with the sweep VEP. Vision Research, 37, 2057-2072.

Kelly, D. H., \& Magnuski, H. S. (1975). Pattern detection and the two-dimensional fourier transform: Circular targets. Vision Research, 15, 911-915.

Kiorpes, L., \& Movshon, J. A. (1998). Peripheral and central factors limiting the development of contrast sensitivity in macaque monkeys. Vision Research, 38, 61-70.

Merigan, W. H. (1996). Basic visual capacities and shape discrimination after lesions of extrastriate area V4 in macaques. Visual Neuroscience, 13, 51-60.

Norcia, A. M., \& Tyler, C. W. (1985). Spatial frequency sweep VEP: Visual acuity during the first year of life. Vision Research, 25, 1399-1408.

Peterzell, D. H., Werner, J. S., \& Kaplan, P. S. (1995). Individual differences in contrast sensitivity functions: Longitudinal study of 4-, 6- and 8-monthold human infants. Vision Research, 35, 961-979.

Richman, J. E., \& Lyons, S. (1994). A forced choice procedure for evaluation of contrast sensitivity function in preschool children. Journal of the American Optometric Association, 65, 859-864. 
Santos, N. A., França, V. C. R. M. \& Cruz, E. D. N. (2007). Sensibilidade ao contraste em crianças 263

Santos, N. A., \& Simas, M. L. B. (2002). Percepção e processamento visual da forma em humanos: Filtros de freqüências radiais de 1 e $4 \mathrm{cpg}$. Psicologia: Reflexão e Crítica, 15, 383-391.

Santos, N. A., Simas, M. L. B., \& Nogueira, R. M. T. B. L. (2004). Processamento visual da forma em humanos: Curvas de limiar de contraste para padrões circularmente simétricos. Psicologia: Reflexão e Crítica, 17, 287-294.

Santos, N. A., Nogueira, R. M. T. B. L., \& Simas, M. L. B. (2005). Processamento visual da forma: Evidências para canais múltiplos de freqüências angulares em humanos. Psicologia: Reflexão e Crítica, 18, 98-103.

Santos, N. A., Oliveira, A. B., Nogueira, R. M. T. B. L., \& Simas, M. L. B. (2006). Mesopic radial frequency contrast sensitivity function for young and older adults. Brazilian Journal of Medical and Biological Research, 39, 791-794.

Simas, M. L. B., \& Santos, N. A. (2002). Contrast sensitivity to radial frequencies modulated by $\mathrm{Jn}$ and jn bessel profiles. Brazilian Journal of Medical and Biological Research, 35, 13571366.

Simas, M. L. B., Santos, N. A., \& Thiers, F. A. (1997). Contrast sensitivity to angular frequency stimuli is higher than that for sinewave gratings in the respective middle range. Brazilian Journal of Medical and Biological Research, 30, 633-636.

Van Sluyters, R. C., Atkinson, M. S., Held, R. M., Hoffman, K., \& Shatz, C. J. (1990). The development of vision and visual perception. In $\mathrm{S}$. W. Spillmann \& J. S. Werner (Eds.), The neurophysiological foundations (pp. 349-379). New York: Academic Press.

Wetherill, G. B., \& Levitt, H. (1965). Sequential estimation of points on a psychometric function. The British Journal of Mathematical and Statistical Psychology, 18, 1-10.
Wilkinson, F., James, T. W., Wilson, H. R., Gati, J. S., Menon, R. S., \& Goodale, M. A. (2000). An fMRI study of the selective activation of human extrastriate form vision areas by radial and concentric gratings. Current Biology, 10, 14551458

Wilson, H. R. (1988). Development of spatiotemporal mechanisms in infant vision. Vision Research, 28, 611-628.

Wilson, H. R., \& Wilkinson, F. (1998). Detection of global structure in glass patterns: Implications for form vision. Vision Research, 38, 2933-2947.

Wilson, R. W., Wilkinson, F., \& Asaad, W. (1997). Concentric orientation summation in human form vision. Vision Research, 37, 2325-2330.

Yuodelis, C., \& Hendrickson, A. (1986). A qualitative and quantitative analysis of the human fovea during development. Vision Research, 26, 847-855.

Apoio Financeiro: Pesquisa financiada pelo $\mathrm{CNPq}$ através de Auxílio Individual (processo: 477258/2001-8), Bolsa IC/PIBIC/UFPB/CNPq e Bolsa de Produtividade em Pesquisa (processo: 307182/2003-7).

Artigo recebido em 31/01/2007.

Aceito para publicação em 07/08/2007.

Endereço para correspondência:

Natanael Antonio dos Santos. Laboratório Percepção, Neurociências e Comportamento (LPNeC-UFPB) Depto. de Psicologia, CCHLA - UFPB. Campus I, Cidade Universitária, CEP 58051-900. João Pessoa-PB. E-mail: natanael.santos@pesquisador.cnpq.br

Natanael Antonio dos Santos é Doutor em Neurociências e Comportamento pela Universidade de São Paulo, Professor Adjunto III do Departamento 
264 Paidéia, 2007, 17(37), 255-264

de Psicologia da UFPB, Bolsista de Produtividade em Pesquisa do CNPq e Coordenador do Laboratório Percepção, Neurociências e Comportamento (LPNeC-UFPB).

Valtenice de Cássia Rodrigues de Matos França é graduada em Psicologia pela UFPB e mestranda em Psicologia Experimental pelo Instituto de Psicologia da Universidade de São Paulo, Bolsista de Mestrado FAPESP.

Éllen Dias Nicácio da Cruz é graduada em Psicologia pela UFPB e mestranda em Psicologia pela UFPE. 